\title{
Rayleigh Noise Mitigated 70-km-Reach Bi-directional WDM-PON with 10-Gb/s Directly Modulated Manchester- duobinary as Downstream Signal
}

\author{
Zhixin Liu ${ }^{*}, 1$, Jing $\mathrm{Xu}^{2}$, Qike Wang ${ }^{1}$ and Chun-Kit Chan ${ }^{1}$ \\ 1.Department of Information Engineering, The Chinese University of Hong Kong, Shatin, N.T., Hong Kong SAR, China. \\ 2. Chair for Communications, University of Kiel, Kaiserstraße 2, D-24143 Kiel, Germany. \\ Tel: +852-2609-8385,Fax:+852-2603-5032,Email:lzx009@ie.cuhk.edu.hk
}

\begin{abstract}
A 70-km-reach bi-directional WDM-PON with 10-Gb/s downstream Manchesterduobinary signal and $1.25-\mathrm{Gb} / \mathrm{s}$ upstream re-modulated NRZ-OOK signals is demonstrated, with effective mitigation of Rayleigh backscattered noise.

OCIS codes: (060.2330) Fiber optics communications; (060.4080) Modulation; (290.1350) Backscattering
\end{abstract}

\section{Introduction}

The wavelength division multiplexed passive optical network (WDM-PON) is an attractive technology to meet the ever-increasing bandwidth demand from enterprises and households. One practical and cost-effective approach is to employ centralized light sources (CLS) at the optical line terminal (OLT) and directly re-use the downstream light as the upstream carrier at the optical network units (ONUs) [1]. Recently, long-reach bi-directional WDM-PON is gaining attention because it is capable of linking the end customers directly to the core networks, thus further reduces the bandwidth transport costs [2]. Therefore, it is desirable to develop CLS long-reach bi-directional WDMPON with cost-effective components. In such single-fiber full-duplex wavelength-reused transmission system, a critical issue that needs to be addressed is how to mitigate the impairments arisen from optical beat noise (OBN) induced by possible back-reflections and Rayleigh backscattering (RBS) for both upstream and downstream signals.

To mitigate the impact of RBS in CLS PONs, different modulation formats have been considered, so as to reduce the spectral overlap between the signal and the interferer noise. In [3], it has shown that downstream differential phase shift keyed (DPSK) signal, together with upstream subcarrier (SCM) signal could achieve 115-km bidirectional transmission. However, it required an expensive high-bandwidth modulator to facilitate upstream data re-modulation and an optical delay interferometer (DI) to demodulate the downstream signal, thus increased the cost of the ONU. A cost-effective solution was proposed to employ Manchester-coded downstream signal, such that the OBN for upstream could be filtered out with a proper low pass filter (LPF) [4]. However, as the Manchester-coded signal has twice the bandwidth than the non-return-to-zero (NRZ) signal, its dispersion tolerance is small. Thus, it cannot support 10-Gb/s downstream signals in CLS long-reach PONs.

In this paper, we propose and demonstrate a cost-effective CLS 70-km-Reach full-duplex WDM-PON with downstream 10-Gb/s Manchester-duobinary signal and upstream 1.25-Gb/s re-modulated NRZ-OOK signal. The downstream pre-chirped Manchester-duobinary signal is generated by directly modulating a chirp managed laser (CML) with an electrical Manchester coded signal, at the OLT [5]. Upstream remodulation is realized via a reflective semiconductor optical amplifier (RSOA), resided at the ONU. The CML-based downstream transmitter offers many advantages to cost-sensitive metro/access applications, including compactness, high output power, and no costly and bulky external modulators required [6]. The reflected OBN in both downstream and upstream paths are mitigated by the electrical low-pass filter (LPF) and high-pass filter (HPF) at the OLT and the ONU, respectively. The experimental results show that the dispersion-tolerant Manchester-duobinary signal can well support data remodulation and OBN suppression in bi-directional transmission.

\section{Proposed system architecture}

Fig. 1 illustrates the proposed WDM-PON architecture utilizing Manchester-duobinary as the downstream signal format. Each transceiver at the OLT generates its downstream Manchester-duobinary signal and receives its upstream re-modulated signals from its respective subscriber. At the OLT, Manchester-coded electrical downstream data is directly modulated onto a designated wavelength channel through a CML. By properly adjusting the modulation current and the central wavelength of the optical spectrum reshaper (OSR) integrated in the CML, 10$\mathrm{Gb} / \mathrm{s}$ Manchester-duobinary signal is generated [5]. To achieve optimum performance for the re-modulated upstream signal, pre-chirping is introduced to the downstream Manchester-duobinary signal by offsetting the OSR. All downstream modulated wavelengths are multiplexed, via an array waveguide grating (AWG), before being fed into an erbium-doped fiber amplifier (EDFA) to boost up the transmitted power. Then the downstream wavelengths are delivered to their respective destined ONUs, via 50-km feeder fiber and 20-km distribution fiber. 

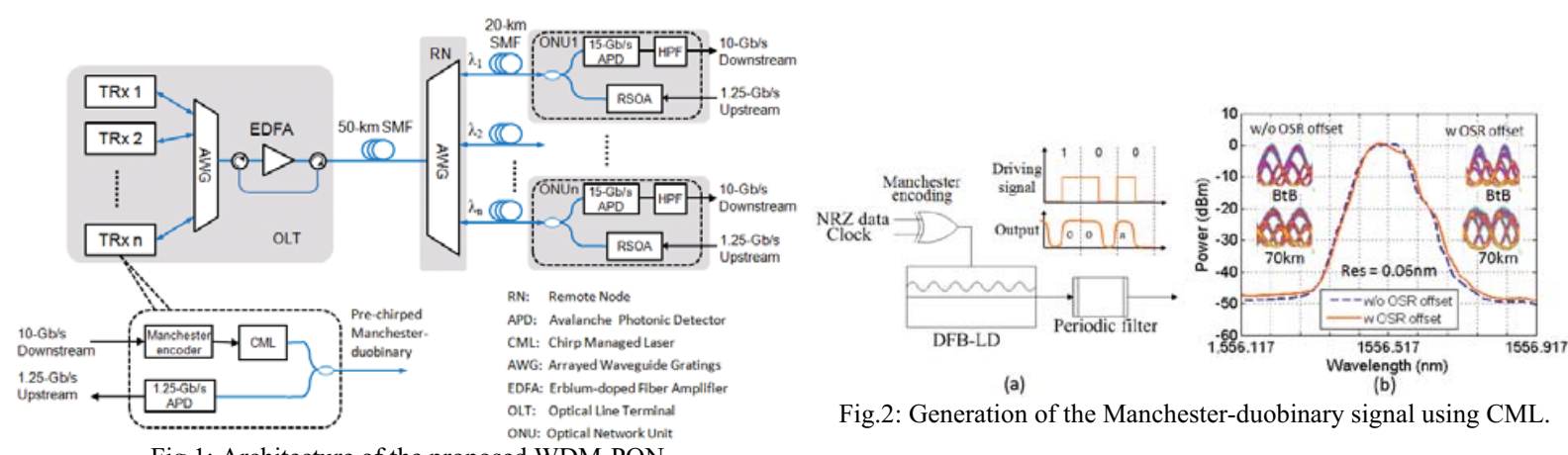

Fig.2: Generation of the Manchester-duobinary signal using CML.

Fig.1: Architecture of the proposed WDM-PON

At each ONU, part of the received downstream signal power is tapped off for detection, while the remaining power is fed into a RSOA for upstream data re-modulation. The finite optical power in each bit of the received downstream signal provides the light source for the $1.25-\mathrm{Gb} / \mathrm{s}$ upstream NRZ-OOK data transmission, before being transmitted back to the OLT, via the same fiber link. A 15-Gb/s avalanche photo diode (APD) and a HPF are used to receive the signal and avoid the Rayleigh backscattering induced performance degradation on the downstream signal. At the OLT, the upstream signal is restored by a $1.25-\mathrm{Gb} / \mathrm{s}$ APD receiver and a LPF. As the downstream induced RBN spreads in higher frequency, LPF can filter out the OBN and recover the upstream signal.

The CML-based Manchester-duobinary transmitter, which comprises an electrical XOR gate and a CML, is schematically shown in Fig. 2(a). Standard Manchester-duobinary exhibited a relatively flat top spectrum. In our experiment, we have introduced pre-chirping to the CML-based Manchester-duobinary signal, via blue-shifting the OSR by about $2 \mathrm{GHz}$. With pre-chirping, the eye diagram obtained at ONU exhibited the minimal overshoot. This not only increased the downstream receiver sensitivity but also improved the signal quality for the re-modulated upstream signal. The optical spectra and the eye diagrams with and without OSR offset were compared in Fig. 2(b).

\section{Experimental setup}

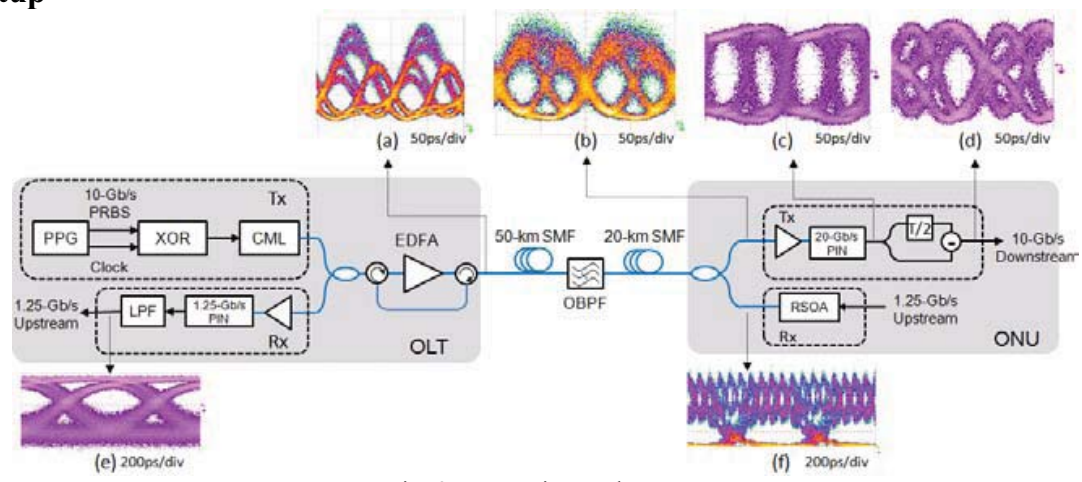

Fig. 3: Experimental setup

The feasibility of the proposed system has been experimentally assessed on one particular channel for proof-ofconcept demonstration. As illustrated in Fig. 3, a standard CML module (Finisar DM200-01) was used in our experiment. The CML at $1556.51 \mathrm{~nm}$ was biased at $75 \mathrm{~mA}$ and modulated by the electrical Manchester coded signal with pseudorandom binary sequence (PRBS) and a word length of $2^{15}-1$. The $V_{p p}$ of the driving signal was 1.18 volts. The output signal was then fed into the EDFA and amplified to $2.3 \mathrm{dBm}$. The transmission link consisted of $50-\mathrm{km}$ feeder fiber and 20-km distribution fiber. An optical bandpass filter (OBPF) with 3-dB bandwidth of $1.28 \mathrm{~nm}$ was used to emulate the RN. At the ONU, half of the received optical power was tapped off for downstream signal reception, while the other half was amplified and re-modulated by a RSOA. The RSOA was biased at $65 \mathrm{~mA}$ and modulated by an electrical NRZ signal with $V_{p p}$ of 4 volts. The downstream optical power input into the RSOA was $-18.6 \mathrm{dBm}$ and the upstream power launched into the fiber link was $0.13 \mathrm{dBm}$. The re-modulated upstream signal was sent back to OLT, via the same transmission link, and was restored by a photo-detector (PD), followed by a $5^{\text {th }}$ order electrical low pass filter (LPF) with 3-dB bandwidth of $798 \mathrm{MHz}$. The downstream signal was received by a $20-\mathrm{Gb} / \mathrm{s}$ photodiode, followed by a HPF constructed by a half-bit electrical delay line and a subtractor. The insets of Fig. 3 show the eye diagrams at various strategic points. The colored eye diagrams were the optical eye diagrams, while the grayed ones were the electrical ones. Clear eye opening for both downstream and upstream signals were obtained with the help of the HPF and the LPF. 


\section{Results and discussion}

Figs. 4(a) and (b) show the measured bit-error-rate (BER) curves for both the downstream and the upstream signals. Error free transmission was achieved after 70-km standard single-mode fiber (SSMF) transmission without dispersion compensation. The BtB sensitivity (at BER of $10^{-9}$ ) for the $10-\mathrm{Gb} / \mathrm{s}$ downstream Manchester-duobinary signal and the $1.25-\mathrm{Gb} / \mathrm{s}$ upstream re-modulated NRZ-OOK signal were measured to be $-27.05 \mathrm{dBm}$ and -32.97 $\mathrm{dBm}$, respectively. After $70-\mathrm{km}$ transmission, their respective sensitivities were measured to be $-28.37 \mathrm{dBm}$ and $32.10 \mathrm{dBm}$, giving -1.05- $\mathrm{dB}$ and 0.87-dB penalty. Without HPF, error free downstream transmission could not be attained. Fig. 4(c) compares the upstream receiver sensitivity for different downstream light sources, including continuous wave $(\mathrm{CW})$ and Manchester-duobinary signal, at different transmission distances. Manchester-duobinary downstream signal outstood the $\mathrm{CW}$ case by achieving 70-km bi-directional transmission. When CW re-modulation was used, error-free upstream transmission over $50 \mathrm{~km}$ could not be achieved, due to the low frequency OBN.

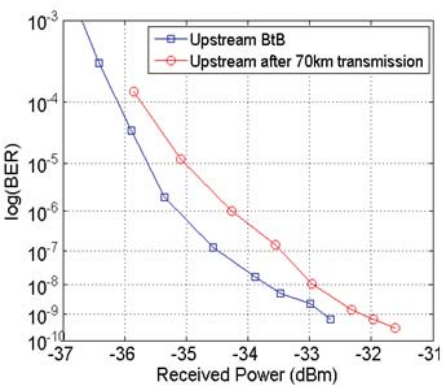

(a)

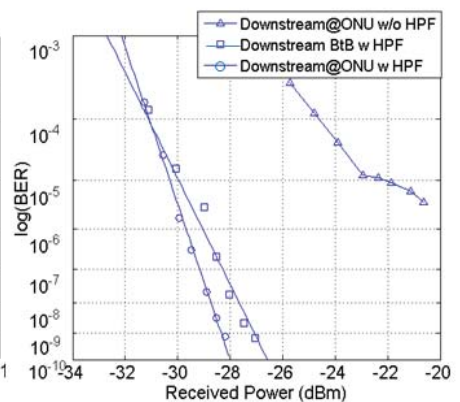

(b)

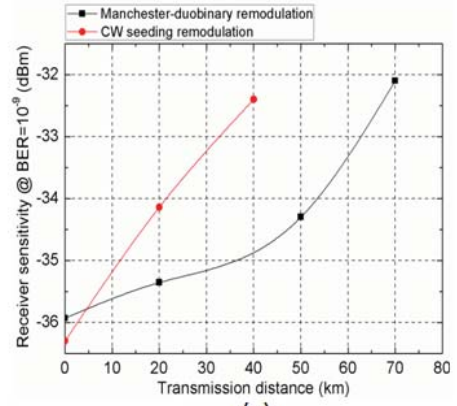

(c)

Fig. 4 BER measurements of (a) the 1.25-Gb/s upstream signal and (b) the 10-Gb/s downstream signal. (c) Comparison of upstream sensitivity for re-modulating $\mathrm{CW}$ seeding and Manchester-duobinary downstream signal.

Fig. 5 (a) and (b) show the electrical spectrum for both upstream and downstream signals with and without the electrical filter. As shown in Fig.5 (a), the spectrum of OBN for upstream signal lied within the high frequency region, attributed to the Manchester-duobinary format of the backscattered downstream signal. Therefore the upstream signal could be recovered by using the LPF to suppress the OBN. Similarly, the downstream signal could be restored by using the HPF, as the filtering of the low-frequency OBN would not affect the downstream signal.

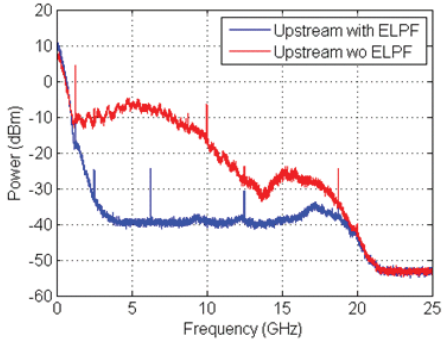

(a)

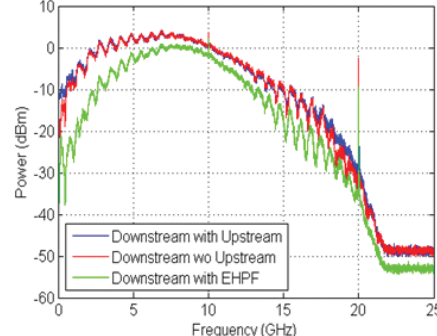

(b)

Fig. 5: Measured RF spectra for (a) upstream and (b) downstream paths with and without LPF and HPF. (Res: $10 \mathrm{MHz}$

\section{Summary}

We have experimentally demonstrated and characterized a 70-km-reach CLS bi-directional WDM-PON. By employing proper electrical filters and the dispersion tolerant Manchester-duobinary downstream signal generated by a CML, $10-\mathrm{Gb} / \mathrm{s}$ downstream and $1.25-\mathrm{Gb} / \mathrm{s}$ upstream transmissions over $70 \mathrm{~km}$ have been realized.

\section{References}

[1] W. Hung, C. K. Chan, L. K. Chen, and F. Tong, "An optical network unit for WDM access networks with downstream DPSK and upstream remodulated OOK data using injection-locked FP laser”, IEEE Photon. Technol. Lett., vol. 15, no. 10, pp. 1992-1994, Oct. 2003.

[2] S. M. Lee, M. H. Kim, and C. H. Lee, "Demonstration of a bi-directional 80-km-reach DWDM-PON with 8-Gb/s capacity," IEEE Photon. Technol. Lett., vol. 19, no. 6, pp. 405-408, Mar. 15, 2007.

[3] A. Chowdhury, H-C. Chien, M-F. Huang, Jianjun Yu, and G-K. Chang, "Rayleigh backscattering noise-eliminated 115-km long-reach bidirectional centralized WDM-PON with $10-\mathrm{Gb} / \mathrm{s}$ DPSK downstream and remodulated 2.5-Gb/s OCS-SCM upstream signal", IEEE Photon. Tech. Lett., vol. 20, no. 24, pp. 2081-2083, 2008.

[4] A. Murakami, Y. J. Lee, K. Y. Cho, Y. Takushima, A. Agata, K. Tanaka, Y. Horiuchi and Y. C. Chung, "Enhanced reflection tolerance of upstream signal in RSOA-based WDM-PON using Manchester coding”, Tech. Dig. of APOC, paper 6783-87, Wuhan, China, 2007.

[5] Z.X. Liu and C.K. Chan, "Generation of dispersion tolerant manchester-duobinary signal using directly-modulated chirp managed laser," IEEE Photon. Technol. Lett., vol. 23, no. 15, pp. 1043-1045, Aug. 2011.

[6] Y. Matsui, D. Mahgerefteh, X. Zheng, C. Liao, Z. F. Fan, K. McCallion, and P. Tayebati, "Chirp-managed directly modulated laser (CML)," IEEE Photon. Technol. Lett., vol. 18, no. 2, pp. 385-388, Jan. 2006. 